\title{
Changes in human gut microbiota influenced by probiotic fermented milk ingestion
}

\author{
Tatsuya Unno, * Jung-Hye Choi,† Hor-Gil Hur,ł Michael J. Sadowsky,§ Young-Tae Ahn,\# Chul-Sung Huh,॥ \\ Geun-Bae Kim, $\prod^{1}$ and Chang-Jun Cha† ${ }^{1}$ \\ *Faculty of Biotechnology, College of Applied Life Sciences, Jeju National University, Jeju 690-756, Republic of Korea \\ †Department of Systems Biotechnology, Chung-Ang University, Anseong 456-756, Republic of Korea \\ †School of Environmental Science and Engineering, Gwangju Institute of Science and Technology, Gwangju 500-712, Republic of Korea \\ §Department of Soil, Water, and Climate; and BioTechnology Institute, University of Minnesota, St. Paul 55108 \\ \#R\&BD Center, Korea Yakult Co. Ltd., Yongin 446-901, Republic of Korea \\ IIInstitute of Green Bio Science and Technology, Graduate School of International Agricultural Technology, Seoul National University, \\ Pyeongchang 232-916, Republic of Korea \\ TDepartment of Animal Science and Technology, Chung-Ang University, Anseong 456-756, Republic of Korea
}

\begin{abstract}
We investigated the effect of consuming probiotic fermented milk (PFM) on the microbial community structure in the human intestinal tract by using highthroughput barcoded pyrosequencing. Six healthy adults ingested 2 servings of PFM daily for $3 \mathrm{wk}$, and their fecal microbiota were analyzed before and after 3 wk of PFM ingestion period and for another 3 wk following the termination of PFM ingestion (the noningestion period). Fecal microbial communities were characterized by sequencing of the V1-V3 hypervariable regions of the $16 \mathrm{~S}$ rRNA gene. All subjects showed a similar pattern of microbiota at the phylum level, where the relative abundance of Bacteriodetes species increased during the PFM ingestion period and decreased during the noningestion period. The increase in Bacteroidetes was found to be due to an increase in members of the families Bacteroidaceae or Prevotellaceae. In contrast to PFM-induced adaptation at the phylum level, the taxonomic composition at the genus level showed a considerable alteration in fecal microbiota induced by PFM ingestion. As revealed by analysis of operational taxonomic units (OTU), the numbers of shared OTU were low among the 3 different treatments (before, during, and after PFM ingestion), but the abundance of the shared OTU was relatively high, indicating that the majority $(>77.8 \%)$ of total microbiota was maintained by shared OTU during PFM ingestion and after its termination. Our results suggest that PFM consumption could alter microbial community structure in the
\end{abstract}

Received October 6, 2014.

Accepted March 11, 2015.

${ }^{1}$ Corresponding authors: kimgeun@cau.ac.kr and cjcha@cau.ac.kr gastrointestinal tract of adult humans while maintaining the stability of microbiota.

Key words: probiotic fermented milk, gut microbiota, pyrosequencing

\section{INTRODUCTION}

Fermented milk products have been consumed by many civilizations over centuries based on belief in their beneficial effects on human health. Yogurt is a coagulated dairy product from the fermentation of lactose in milk by the traditional yogurt starter cultures, such as Lactobacillus delbrueckii ssp. bulgaricus and Streptococcus thermophilus (Adolfsson et al., 2004). These bacteria are called probiotics, traditionally defined as viable microorganisms that offer beneficial effects when ingested by individuals (Rolfe, 2000) and have been used to enhance gut health for centuries. Some lactic acid bacteria, such as bifidobacteria and lactobacilli, have been combined with yogurt starters because of their probiotic properties. Probiotics are frequently included in yogurts, often together with some prebiotic compounds. Nondigestible food ingredients such as oligosaccharides, dietary fiber, and lactulose are generally used as prebiotics. Together, this combination of dietary supplements is referred to as a synbiotic yogurt (Palaria et al., 2012). Although these products have been used by many people, the mechanism(s) by which they act have yet to be identified (Bisanz and Reid, 2011).

The human gut microbiota contains at least 100 times as many genes as the human genome (Gill et al., 2006) and provides important human physiological functions such as glycan biosynthesis and metabolism (Gill et al., 2006; Sanz et al., 2008). Although a core human gut microbiota has been reported (Caporaso et al., 2011; Hunt et al., 2011), dietary habits can alter the com- 
position of the gut microbiota to change the metabolic potential (Hildebrandt et al., 2009). Consumption of yogurt has been proposed as a means to prevent obesity (Sun and Zemel, 2004; Diepvens et al., 2007) and to improve intestinal function in the gastrointestinal (GI) tract of some humans (Anukam et al., 2008; Schiffrin et al., 2009; Nikooyeh et al., 2011).

Although colonization in the gut by beneficial microorganisms may have significant effects on human health (Larsen et al., 2010) and may change the metabolic capacity of the gut (Claus et al., 2011), many probiotic bacteria are unable to colonize the human gut (Kullen et al., 1997) and pass through the human GI tract (Elli et al., 2006). Previously, McNulty et al. (2011) observed no changes in the abundance of a probiotic strain, Bifidobacterium animalis, in either humans or mice after yogurt ingestion, yet they did report definitive changes in the microbiota's metabolic pathways, such as increases in carbohydrate and nucleotide metabolism and decreases in amino acid and lipid metabolism. In addition, the effects of yogurt ingestion were observed with heat-sterilized yogurt (García-Albiach et al., 2008) as well as dry heat-killed probiotic powder (Shin et al., 2010; Vintiñi and Medina, 2011). These studies suggest that probiotics may indirectly influence gut microbiota without colonizing the human intestinal tract. Although there has been no direct evidence of the mechanisms for how probiotic bacteria can alter gut microbiota, secretion of antimicrobial compounds such as bacteriocins by some probiotic strains is known to prevent bacterial adhesion and evasion of epithelial cells (Gerritsen et al., 2011). Metabolic modeling of species interaction in the human microbiome predicts potential competition and syntrophy (Levy and Borenstein, 2013).

In many studies, culture-based and molecular-based approaches that target specific groups of bacteria have been used to analyze changes in the gut microbiota upon various treatments (Elli et al., 2006; Schiffrin et al., 2009; Saxelin et al., 2010). Recently, high-throughput sequencing technologies such as pyrosequencing have been used to analyze microbial community composition and dynamics from diverse environments, and these approaches offer an opportunity to understand the whole microbial community much more comprehensively than traditional culture-based approaches (Claesson et al., 2009; Griffen et al., 2012; Osei-Poku et al., 2012). In this study, rather than studying the influence of specific probiotic strains on gut microbiota, we used highthroughput deep sequencing to investigate whether consumption of probiotic fermented milk (PFM) could result in alterations in the microbial community structure of human gut microbiota at different phylogenetic levels.

\section{MATERIALS AND METHODS}

\section{In Vivo Experiment}

Six healthy female volunteers, 20 to 24 yr of age, participated in this study (Supplemental Table S1; http:// dx.doi.org/10.3168/jds.2014-8943). During the study period, the volunteers were asked to maintain their usual lifestyle and diet but to refrain from consuming any drugs and fermented milk products other than those provided by us. Written consent was obtained from each person, and the study was approved by the Research Ethics Committee of Chung-Ang University. The PFM was prepared using fresh milk, skim milk powder, sugar, and a stabilizer, and was fermented with the ABCT-BH starter culture (Culture System Inc., Mishawaka, IN) containing Lactobacillus acidophilus CSG (Han et al., 2005b), Lactobacillus brevis HY7401 (Lee et al., 2008), Bifidobacterium longum HY8001 (Han et al., 2005a), Lactobacillus casei HY2782, and Streptococcus thermophilus. The PFM was also supplemented with a dietary fiber mixture and lactulose as prebiotic components. The final preparation $(140 \mathrm{~mL}$ serving size) contained dietary fiber ( $3 \mathrm{~g})$, lactulose (1 g), nonfat milk solids $(8.5 \%)$, protein (3.2 g), fat $(3.0 \mathrm{~g})$, and Lactobacillus $\left(1.0 \times 10^{8} \mathrm{cfu} / \mathrm{mL}\right)$. The volunteers consumed 2 servings of PFM daily (one after breakfast and the other after dinner) for $3 \mathrm{wk}$, and then stopped consuming PFM for an additional $3 \mathrm{wk}$ of the study period (Supplemental Figure S1; http://dx.doi. org/10.3168/jds.2014-8943). Fecal samples were collected from each subject at 3 time points: before PFM ingestion ( $\mathrm{d} 0$ ), at the end of PFM ingestion period (d 21), and another 3 wk following the end of PFM ingestion (d 42). All fecal samples were stored at $-80^{\circ} \mathrm{C}$ until used.

\section{DNA Extraction from Fecal Samples}

Approximately 0.5-g aliquots of feces from each sample were washed twice with $5 \mathrm{~mL}$ of PBS (pH 7.4) and were subjected to DNA extraction using an UltraClean Fecal DNA Kit (Mo Bio Laboratories, Carlsbad, CA). Approximately $100 \mathrm{ng}$ of DNA was used for PCR amplification of the $16 \mathrm{~S}$ rRNA gene in samples.

\section{Amplification of 16S rRNA Gene and Sequencing}

The extracted DNA was amplified using primers targeting the V1 and V3 hypervariable regions of the bacterial $16 \mathrm{~S}$ rRNA gene using primers 27F: $5^{\prime}$-XAC-GAGTTTGATCMTGGCTCAG-3' and 518R: 5'-X-AC-WTTACCGCGGCTGCTGG-3', where X de- 
notes an 8-nucleotide-long barcode uniquely designed for each DNA sample followed by a linker AC, and $\mathrm{M}$ and $\mathrm{W}$ bases indicate mixed-base composition for $\mathrm{A}$ or $\mathrm{C}$ and $\mathrm{A}$ or $\mathrm{T}$, respectively. The $\mathrm{PCR}$ reactions were performed under the following conditions: initial denaturation at $94^{\circ} \mathrm{C}$ for $5 \mathrm{~min}$, followed by 25 cycles of denaturation at $94^{\circ} \mathrm{C}$ for $30 \mathrm{~s}$, annealing at $60^{\circ} \mathrm{C}$ for 30 $\mathrm{s}$, and elongation at $72^{\circ} \mathrm{C}$ for $80 \mathrm{~s}$. The amplified PCR products were purified using a purification kit (Qiagen, Valencia, CA), and $1 \mu \mathrm{g}$ of PCR product from each sample was pooled and subjected to pyrosequencing. Sequencing was carried out by ChunLab Inc. (Seoul, South Korea) using a GS FLX Titanium 454 Sequencing System (Roche, South San Francisco, CA) according to the manufacturer's instructions.

\section{Pyrosequencing Data Processing}

Pyrosequencing outputs were processed according to the Schloss standard operation procedure (Schloss et al., 2011), using Mothur version 1.31.1 (Schloss et al., 2009). Briefly, all pyrosequencing reads were processed for primer sequence trimming, alignable consensus region screening, and removal of chimeric and cyanobacteria-derived sequences. Alignment was done by using the RDP (Ribosomal Database Project) aligner (Nawrocki et al., 2009). Taxonomic classification was performed, at an $80 \%$ cut-off, using Mothur with an RDP training set. All processed reads were normalized by making a subset of samples using the Mothur sub.sample routine, based on the minimum number of reads obtained for a sample. As recommended previously (Schloss and Westcott, 2011), operational taxonomic units (OTU) were assigned based on analysis conducted at a distance of 0.03, using Mothur average clustering. Analyses based on phylogenetic trees were carried out using the online FastUnifrac (Hamady et al., 2010) tool. For the FastUnifrac analysis, a Newick format tree file was generated from the Mothur-processed FASTA file by FastTree software (Price et al., 2009). The ID mapping files were also created from Mothur-processed "name" and "group" files by using a PERL script (available from the authors). Weighted UniFrac analysis with normalized option was applied as recommended (Lozupone et al., 2007).

\section{Statistical Analysis}

Cluster analysis based on Bray-Curtis distance was performed using PAST (version 2.17; Hammer et al., 2001) to measure similarity among samples. Abundant genera ( $>0.1 \%$ relative abundance of each genus) were represented in a heatmap with a dendrogram added to the top. Statistically significant differences among samples were determined by using one-way ANOVA and the Student $t$-test, performed using PAST (version 2.17).

\section{RESULTS AND DISCUSSION}

\section{Evaluation of Pyrosequencing Data}

A total of 223,726 reads were obtained from 18 fecal samples in this study. All 454 sequence data are available from the EMBI-EBI Sequence Read Archive under the study accession number ERP001924 (http://www. ebi.ac.uk/ena/data/view/ERP001924). Mothur has implemented the amplicon noise algorithm developed by Quince et al. (2011), by which homopolymer-derived 454 sequencing errors can be effectively removed. In this study, 3 screening steps (denoising and chimera and cyanobacteria-like sequence removal) were applied. The denoising algorithm step removed 24 to $68 \%$ of the total reads per sample (Supplemental Table S2; http://dx.doi.org/10.3168/jds.2014-8943).The numbers of reads originally obtained and those remaining after processing are listed in Table 1 . The greatest number of reads was obtained from sample 1a (29,047 reads). In contrast, an almost 10-fold-smaller number of reads was obtained from sample $2 \mathrm{c}(2,260$ reads $)$. To normalize analyses based on the minimum number of reads obtained per sample, 2,260 sequences were randomly extracted from each sample set and used for the downstream analyses. This randomization did not bias the original data (Supplemental Figure S2; http://dx.doi. org/10.3168/jds.2014-8943).

\section{Diversity of Microbial Communities}

The diversity indices of the subset samples are listed in Table 1. An average of 0.96 coverage, with a standard deviation of 0.02 , was obtained for total 18 samples examined in this study. After 3 wk of PFM ingestion, the Shannon diversity index, except for individual 6 , significantly decreased $(P<0.05)$, suggesting that species diversity may have been lowered by consumption of PFM. Species richness, as shown in the Chao1 index, varied independently from PFM ingestion. Results from rarefaction curve analysis also tended to show the effects of PFM ingestion on gut microbial community, although no consistent trends were observed (Supplemental Figure S3; http://dx.doi.org/10.3168/ jds.2014-8943).

\section{Changes in Microbial Communities}

As shown in Figure 1A, microbial community shifts at the phylum level were observed in all individuals af- 
Table 1. Number of processed sequences and diversity indices

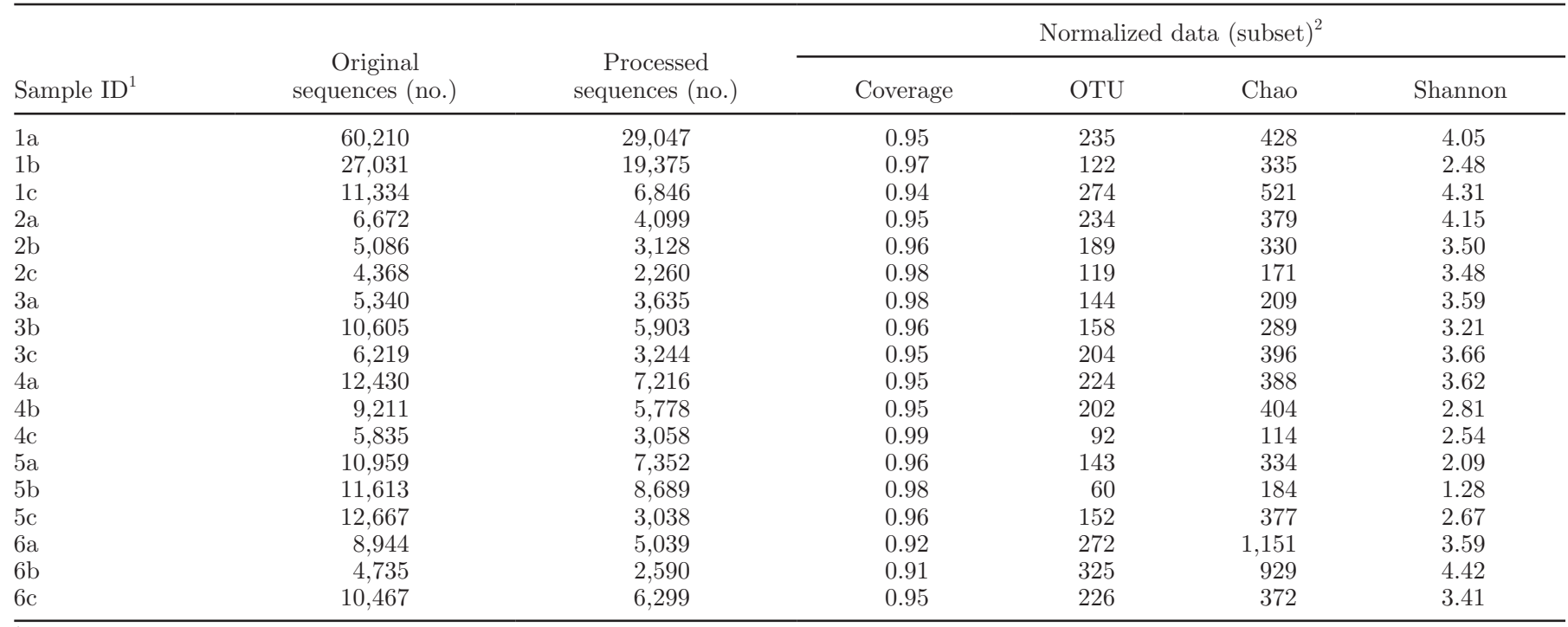

${ }^{1}$ The ID numbers refer to individuals sampled; letters a (before), b (during), and c (after) denote the time of sample collection relative to ingestion of probiotic fermented milk.

${ }^{2} \mathrm{OTU}=$ operational taxonomic unit; Chao $=$ the Chao 1 estimator; Shannon $=$ a Shannon index-based measure of evenness.

ter 3 wk of PFM ingestion and after an additional 3 wk of noningestion. In particular, the relative abundances of the phyla Bacteroidetes and Firmicutes were significantly altered $(P<0.05-0.001$; Figure 1B). Bacteroidetes increased during PFM ingestion and decreased again after the end of ingestion. Firmicutes changed in the opposite way. The ratio of relative abundance of Bacteroidetes and Firmicutes was previously suggested to differentiate lean and obesity types in humans (Ley et al., 2005, 2006). Hildebrandt et al. (2009) reported that these changes are more associated with diet than with genetic differences, and the effects of yogurt on weight control have been reported in previous studies (Sun and Zemel, 2004; Diepvens et al., 2007; White et al., 2009). Although the mechanism(s) by which this occurs are yet to be well understood, our results suggest that bacterial community shifts are likely triggered by PFM ingestion. The possible modulating mechanisms exerted by probiotic strains may include reduction of luminal $\mathrm{pH}$, competition for nutrients, secretion of antimicrobial compounds (bacteriocins), and induction of the production of antimicrobial compounds (defensins) by the host (Gerritsen et al., 2011). Further study is required to establish which component in the PFM can modulate the intestinal microbiota and whether any synergistic effects exist between probiotic strains and prebiotic components included in this PFM.

The shifts in bacterial composition at the phylum level were caused by the changes at the family level (Figure 1C). Two families appeared to contribute to an increase in Bacteroidetes species during the PFM ingestion period (at d 21). Individuals 1, 2, and 6 had large increases in members of the family Bacteroidaceae $(P<0.05)$, whereas individuals 3,4 , and 5 had large increases in members of the family Prevotellaceae $(P<$ 0.05). The decrease in members of Firmicutes during the PFM ingestion period (at d 21) was mainly due to decreases in Ruminococcaceae and Lachnospiraceae (individuals $1,2,4$, and $5 ; P<0.05$ ), although individuals 3 and 6 showed different changes.

Heatmap analysis showed the relative abundance of genera (>0.1\%; Figure 2). The genera Prevotella, Bacteroides, and Faecalibacterium were the predominant members in the gut microbiota (Figure 2; Supplemental Table S3; http://dx.doi.org/10.3168/jds.2014-8943). Moreover, members of Prevotella and Bacteroides were found to be major contributors producing changes in the corresponding families. As shown in Figure 3, clustering results suggested limited correlation among the samples before and after PFM ingestion and apparent variation in different individuals at the genus level.

UniFrac-based principal coordinate (PC) analysis (PCoA) was conducted to investigate the effects of PFM ingestion on phylogenetic diversity. Results in Figure 3 show that the gut microbial communities shifted downward to PC2/PC3 during PFM ingestion and upward to $\mathrm{PC} 1$ during the noningestion period in all the human samples, except in individual 6 (Figure 3). This consistent microbial community shift that was characterized by PCoA may give insight to the mechanisms by which PFM acts on human intestine; Unifrac-based PCoA has been used in many studies to 


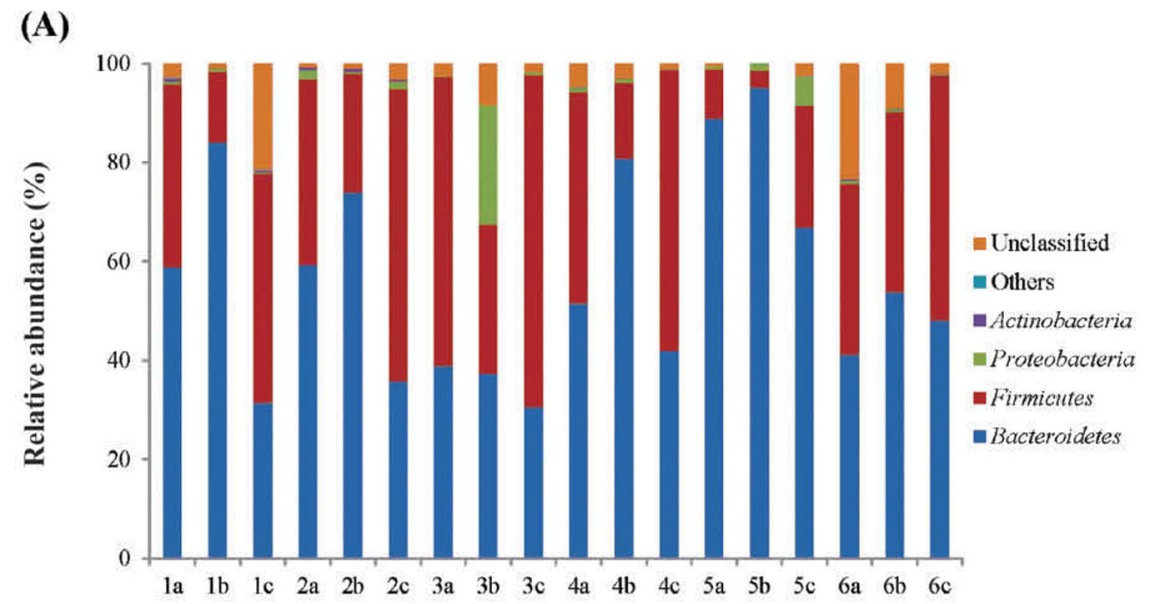

(B)
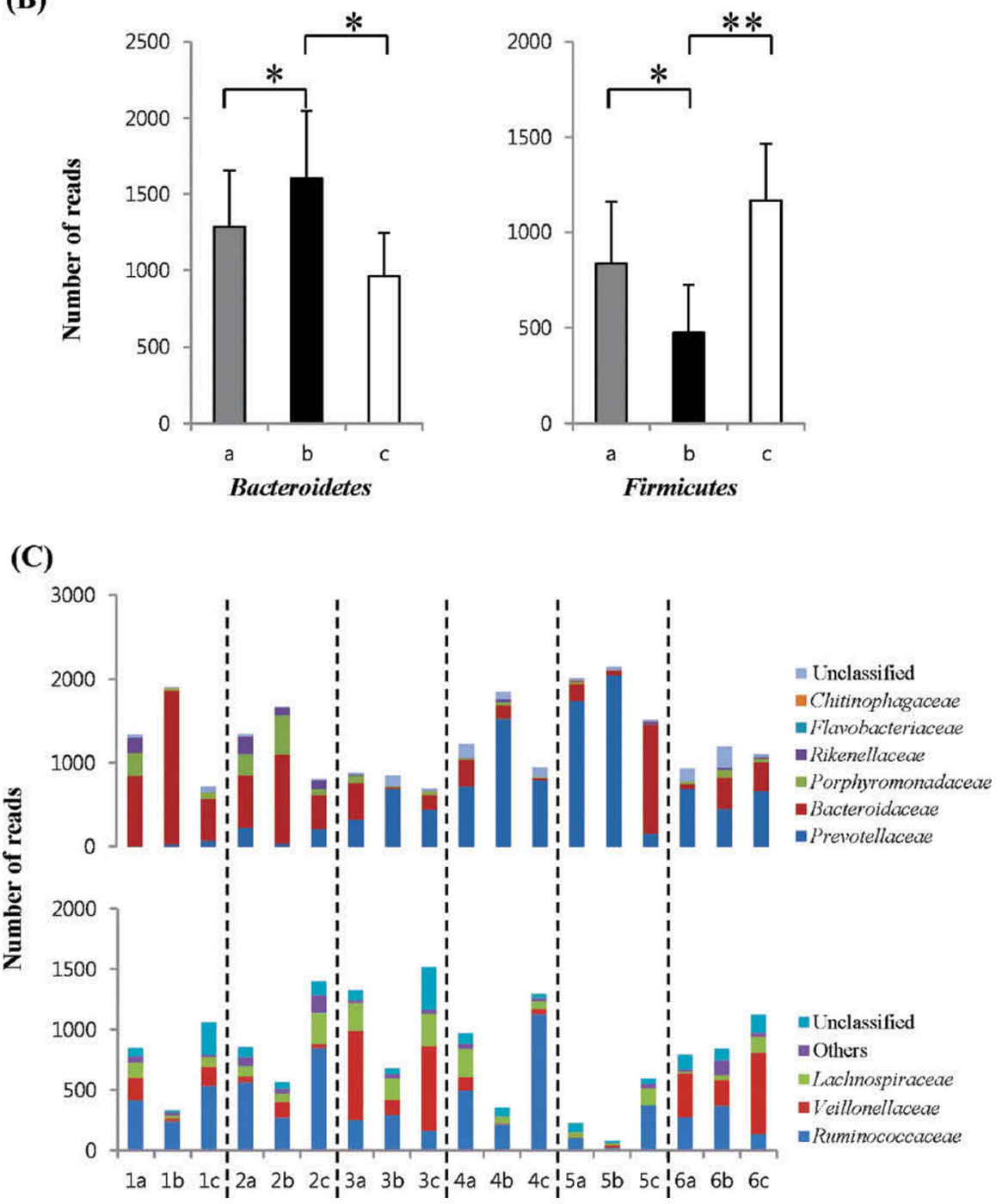

Figure 1. Microbial community changes in feces of each individual collected before, during, and after ingestion of probiotic fermented milk (PFM): (A) bacterial composition at the phylum level; (B) relative abundance of the 2 phyla Bacteroidetes and Firmicutes; (C) bacterial composition of members of Bacteroidetes (upper) and Firmicutes (lower) at the family level. Letters a (before), b (during), and c (after) denote the time of sample collection relative to ingestion of PFM. Mean values $\pm \mathrm{SD}$ are plotted $\left({ }^{*} P<0.05,{ }^{* *} P<0.001\right.$; Student's $t$-test). 

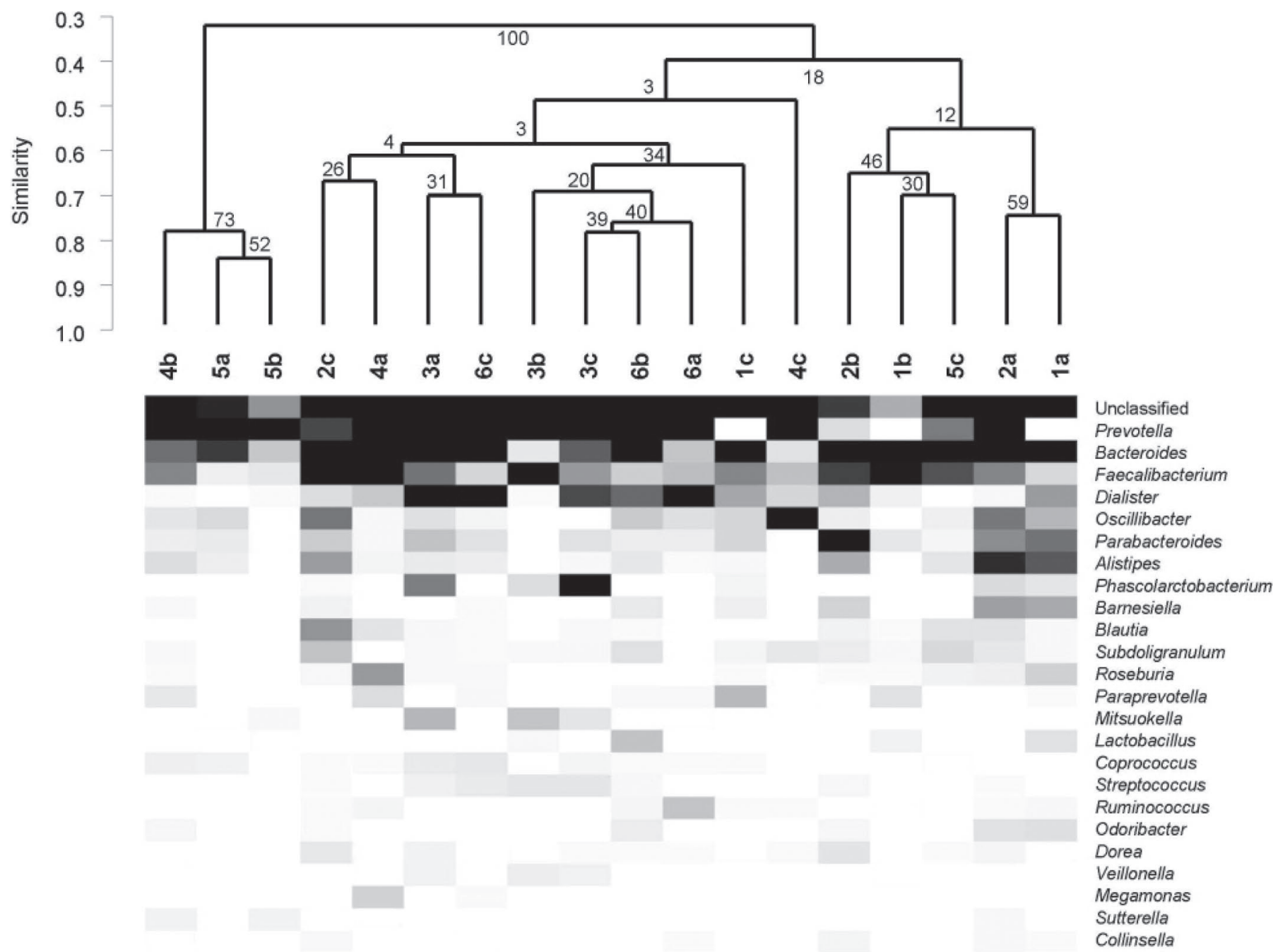

MinScale $=0.00$

MaxScale $=10.00$

Figure 2. Heatmap depicting relative abundance of the most abundant genera $(>0.1 \%)$ arranged by hierarchical clustering of fecal samples. Letters a (before), b (during), and c (after) denote the time of sample collection relative to ingestion of probiotic fermented milk.

characterize human gut microbiota (Momozawa et al., 2011; Nam et al., 2011; Flores et al., 2012; Griffen et al., 2012; Kim et al., 2012; Wang et al., 2012).

\section{Heterogeneity and Stability of Fecal Microbiota}

Bacterial community composition was analyzed based on OTU at a distance of 0.03. The results shown in Table 2 indicated that the bacterial community exhibited both heterogeneity and stability among different treatment samples $(\mathrm{a}=$ before, $\mathrm{b}=$ during, and $\mathrm{c}=$ after PFM ingestion); the number of OTU of a that were shared with a or c was relatively low $(40.9 \%$ of $\mathrm{b}$ and $30.8 \%$ of $\mathrm{c}$ ), suggesting that a remarkable alteration of OTU occurred in the human gut during the whole experimental period. However, these results may overestimate the changes upon each treatment because deep sequencing inflated the numbers of rare OTU and reduced the apparent fraction of shared OTU (Martínez et al., 2013). When the relative abundance of shared OTU was considered, greater percentages of total reads of b and c were shared with a (90.7 and $77.8 \%$, respectively; Table 2 ). In addition, only $16.6 \%$ of OTU of a were maintained through the entire experimental period, but these OTU accounted for nearly half $(47.5 \%)$ of the starting microbiota (Table 2). Our results suggested that the majority of total microbiota was maintained by shared OTU during PFM inges- 


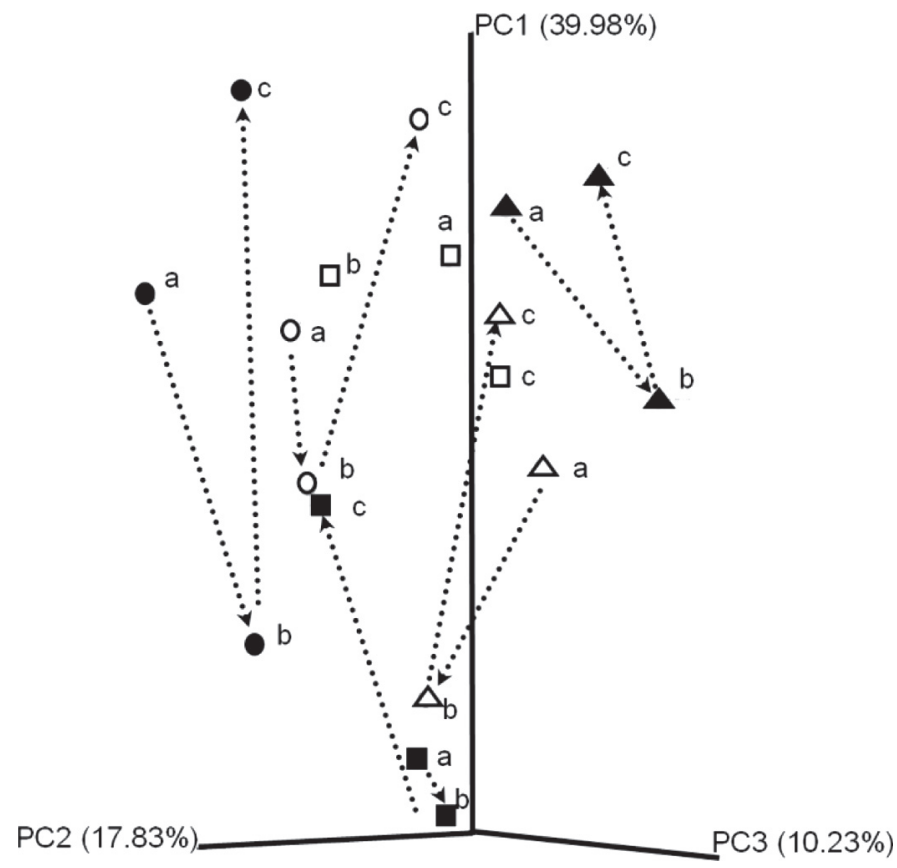

Figure 3. Principal coordinate $(\mathrm{PC})$ analysis of microbial community shifts occurring during probiotic fermented milk (PFM) ingestion and noningestion periods in each individual. Letters a (before), b (during), and c (after) denote the time of sample collection relative to ingestion of PFM; symbols represent each of 6 individuals.

tion and after its termination, although a substantial number of rare OTU appeared or disappeared during the entire period. It was previously reported that a temporal core of 33 to 40 species that account only for $12 \%$ of the total OTU added up to $>75 \%$ of the total sequences over a 1-yr period (Martínez et al., 2013). Various external influences such as diet change, geography, inflammation, stress, and physiological state could cause temporal variations within individuals (Pepper and Rosenfeld, 2012; Tremaroli and Backhed, 2012).

\section{Abundance of Probiotic Strains}

Results from analysis of genus abundance also showed the relative abundance of probiotic strains during and after PFM ingestion. Changes in the relative abundance of the genus Lactobacillus, which was the major probiotic component of PFM, were not consistent among individuals, although ANOVA results showed some degree of statistical significance $(F=3.86, P=0.057)$. Although Saxelin et al. (2010) reported a substantial amount of probiotic strains in feces during yogurt ingestion, we rarely detected members of genus Bifidobacterium, even in a sample with a sufficient sequence depth (19,368 reads; Supplemental Table S4; http:// dx.doi.org/10.3168/jds.2014-8943), although we did so by using culture-dependent assays (data not shown). To improve the detection of bifidobacteria through pyrosequencing, we selected 2 samples ( $3 \mathrm{a}$ and $3 \mathrm{~b}$ ) and conducted pyrosequencing using a Bifidobacteriumspecific forward primer (V1-9F: 5'-X-AC-GGGTTCGATTCTGGCTCAG, where X denotes a barcode), which was added at a ratio of $10 \%$ of the total forward primer concentration. Approximately 8,000 sequences were obtained for each of the 2 samples. Sequences obtained for the 2 samples did not show a significant difference from the original data (Supplemental Figure S4; http://dx.doi.org/10.3168/jds.2014-8943) and bifidobacteria were detected at $<0.05 \%$ abundance. This suggests that there were probably few bifidobacteria present in the gut even after PFM ingestion. The discrepancy observed in this study may be due to the low detection limit of $16 \mathrm{~S}$ rRNA gene universal primers for microbial community analysis compared with that obtained via enrichment plating. Similarly, previous analyses of bovine fecal microbiota by using pyrosequencing failed to detect Escherichia coli strains, although E. coli was successfully isolated from the same feces by plating (Unno et al., 2010). A recent study has shown that use of different variable regions may increase the efficiency of detecting Bifidobacterium species (Sim et al., 2012).

\section{CONCLUSIONS}

Ingestion of PFM led to a consistent microbial community shift as revealed by community composition at the phylum level and Unifrac-based phylogenetic diversity analysis. In contrast, at the lower phylogenetic level, the majority of original gut microbiota was main-

Table 2. The percentage (SD in parentheses) of shared operational taxonomic units (OTU) and their abundance upon ingestion of probiotic fermented milk $(\mathrm{PFM})^{1}$

\begin{tabular}{lcccc}
\hline Item & $\mathrm{a}$ & Shared with b & Shared with c & Shared with b and c \\
\hline Number of OTU & 100 & $40.9^{2}(8.3)$ & $30.8^{2}(10)$ & $16.6^{3}(9.5)$ \\
Read abundance & 100 & $90.7^{4}(6.5)$ & $77.8^{4}(8.7)$ & $47.5^{3}(18.8)$ \\
\hline
\end{tabular}

${ }^{1} \mathrm{a}=$ before, $\mathrm{b}=$ during, and $\mathrm{c}=$ after PFM ingestion.

${ }^{2}$ Number of OTU of a shared with those of b (or c) to the total number of OTU of b (or c).

${ }^{3}$ Number (or reads) of OTU of a shared with both b and $\mathrm{c}$ to the total number (or reads) of OTU of a.

${ }^{4}$ Reads of OTU of a shared with those of b (or c) to the total reads of OTU of b (or c). 
tained after PFM ingestion, whereas only 30 to $40 \%$ of OTU were shared. Our attempt to analyze the changes in gut microbiota upon PFM ingestion indicates that PFM consumption could alter microbial community structure in the human GI tract, simultaneously maintaining the stability of microbiota. Further studies on the effects of individual components of PFM on microbial community should provide a better understanding of correlation between nutrition and gut microbiota of human.

\section{ACKNOWLEDGMENTS}

This work was supported by the Basic Science Research Program through the National Research Foundation of Korea (NRF) funded by the Ministry of Education, Science and Technology (2009-0090020) and partly supported by a grant from Korea Yakult Co. Ltd. (Seoul, South Korea).

\section{REFERENCES}

Adolfsson, O., S. N. Meydani, and R. M. Russell. 2004. Yogurt and gut function. Am. J. Clin. Nutr. 80:245-256.

Anukam, K. C., E. O. Osazuwa, H. B. Osadolor, A. W. Bruce, and G. Reid. 2008. Yogurt containing probiotic Lactobacillus rhamnosus GR-1 and L. reuteri RC-14 helps resolve moderate diarrhea and increases CD4 count in HIV/AIDS patients. J. Clin. Gastroenterol. 42:239-243.

Bisanz, J. E., and G. Reid. 2011. Unraveling how probiotic yogurt works. Sci. Transl. Med. 3:106ps41.

Caporaso, J. G., C. L. Lauber, E. K. Costello, D. Berg-Lyons, A. Gonzalez, J. Stombaugh, D. Knights, P. Gajer, J. Ravel, N. Fierer, J. I. Gordon, and R. Knight. 2011. Moving pictures of the human microbiome. Genome Biol. 12:R50.

Claesson, M. J., O. O'Sullivan, Q. Wang, J. Nikkila, J. R. Marchesi, H. Smidt, W. M. de Vos, R. P. Ross, and P. W. O'Toole. 2009. Comparative analysis of pyrosequencing and a phylogenetic microarray for exploring microbial community structures in the human distal intestine. PLoS ONE 4:e6669.

Claus, S. P., S. L. Ellero, B. Berger, L. Krause, A. Bruttin, J. Molina, A. Paris, E. J. Want, I. de Waziers, O. Cloarec, S. E. Richards, Y. Wang, M. E. Dumas, A. Ross, S. Rezzi, S. Kochhar, P. Van Bladeren, J. C. Lindon, E. Holmes, and J. K. Nicholson. 2011. Colonization-induced host-gut microbial metabolic interaction. MBio 2:e00271-10.

Diepvens, K., S. Soenen, J. Steijns, M. Arnold, and M. WesterterpPlantenga. 2007. Long-term effects of consumption of a novel fat emulsion in relation to body-weight management. Int. J. Obes. (Lond.) 31:942-949.

Elli, M., M. L. Callegari, S. Ferrari, E. Bessi, D. Cattivelli, S. Soldi, L. Morelli, N. Goupil Feuillerat, and J. M. Antoine. 2006. Survival of yogurt bacteria in the human gut. Appl. Environ. Microbiol. 72:5113-5117.

Flores, R., J. Shi, M. H. Gail, P. Gajer, J. Ravel, and J. J. Goedert. 2012. Assessment of the human faecal microbiota: II. Reproducibility and associations of $16 \mathrm{~S}$ rRNA pyrosequences. Eur. J. Clin. Invest. 42:855-863.

García-Albiach, R., M. J. Pozuelo de Felipe, S. Angulo, M. I. Morosini, D. Bravo, F. Baquero, and R. del Campo. 2008. Molecular analysis of yogurt containing Lactobacillus delbrueckii ssp. bulgaricus and Streptococcus thermophilus in human intestinal microbiota. Am. J. Clin. Nutr. 87:91-96.
Gerritsen, J., H. Smidt, G. T. Rijkers, and W. M. de Vos. 2011. Intestinal microbiota in human health and disease: The impact of probiotics. Genes Nutr. 6:209-240.

Gill, S. R., M. Pop, R. T. Deboy, P. B. Eckburg, P. J. Turnbaugh, B. S. Samuel, J. I. Gordon, D. A. Relman, C. M. Fraser-Liggett, and K. E. Nelson. 2006. Metagenomic analysis of the human distal gut microbiome. Science 312:1355-1359.

Griffen, A. L., C. J. Beall, J. H. Campbell, N. D. Firestone, P. S. Kumar, Z. K. Yang, M. Podar, and E. J. Leys. 2012. Distinct and complex bacterial profiles in human periodontitis and health revealed by $16 \mathrm{~S}$ pyrosequencing. ISME J. 6:1176-1185.

Hamady, M., C. Lozupone, and R. Knight. 2010. Fast UniFrac: Facilitating high-throughput phylogenetic analyses of microbial communities including analysis of pyrosequencing and PhyloChip data. ISME J. 4:17-27.

Hammer, Ø., D. A. T. Harper, and P. D. Ryan. 2001. PAST: Paleontological statistics software package for education and data analysis. Paleontol. Electron. 4:1-9.

Han, S. Y., C.-S. Huh, Y.-T. Ahn, K.-S. Lim, Y.-J. Baek, and D.-H. Kim. 2005a. Hepatoprotective effect of lactic acid bacteria. J. Microbiol. Biotechnol. 15:887-890.

Han, S. Y., C.-S. Huh, Y.-T. Ahn, K.-S. Lim, Y.-J. Baek, and D.-H. Kim. 2005b. Hepatoprotective effect of lactic acid bacteria, inhibitors of $\beta$-glucuronidase production against intestinal microflora. Arch. Pharm. Res. 28:325-329.

Hildebrandt, M. A., C. Hoffmann, S. A. Sherrill-Mix, S. A. Keilbaugh, M. Hamady, Y. Y. Chen, R. Knight, R. S. Ahima, F. Bushman, and G. D. Wu. 2009. High-fat diet determines the composition of the murine gut microbiome independently of obesity. Gastroenterology 137:1716-1724.

Hunt, K. M., J. A. Foster, L. J. Forney, U. M. Schutte, D. L. Beck, Z. Abdo, L. K. Fox, J. E. Williams, M. K. McGuire, and M. A. McGuire. 2011. Characterization of the diversity and temporal stability of bacterial communities in human milk. PLoS ONE 6:e21313.

Kim, B. S., J. N. Kim, S. H. Yoon, J. Chun, and C. E. Cerniglia. 2012. Impact of enrofloxacin on the human intestinal microbiota revealed by comparative molecular analysis. Anaerobe 18:310-320.

Kullen, M. J., M. M. Amann, M. J. O'Shaughnessy, D. J. O'Sullivan, F. F. Busta, and L. J. Brady. 1997. Differentiation of ingested and endogenous bifidobacteria by DNA fingerprinting demonstrates the survival of an unmodified strain in the gastrointestinal tract of humans. J. Nutr. 127:89-94.

Larsen, N., F. K. Vogensen, F. W. van den Berg, D. S. Nielsen, A. S. Andreasen, B. K. Pedersen, W. A. Al-Soud, S. J. Sorensen, L. H. Hansen, and M. Jakobsen. 2010. Gut microbiota in human adults with type 2 diabetes differs from non-diabetic adults. PLoS ONE 5:e9085.

Lee, H.-S., S.-Y. Han, E.-A. Bae, C.-S. Huh, Y.-T. Ahn, J.-H. Lee, and D.-H. Kim. 2008. Lactic acid bacteria inhibit proinflammatory cytokine expression and bacteial glycosaminoglycan degradation activity in dextran sulfate sodium-induced colitic mice. Int. Immunopharmacol. 8:574-580.

Levy, R., and E. Borenstein. 2013. Metabolic modeling of species interaction in the human microbiome elucidates community-level assembly rules. Proc. Natl. Acad. Sci. USA 110:12804-12809.

Ley, R. E., F. Backhed, P. Turnbaugh, C. A. Lozupone, R. D. Knight, and J. I. Gordon. 2005. Obesity alters gut microbial ecology. Proc. Natl. Acad. Sci. USA 102:11070-11075.

Ley, R. E., P. J. Turnbaugh, S. Klein, and J. I. Gordon. 2006. Microbial ecology: human gut microbes associated with obesity. Nature 444:1022-1023.

Lozupone, C. A., M. Hamady, S. T. Kelley, and R. Knight. 2007. Quantitative and qualitative beta diversity measures lead to different insights into factors that structure microbial communities. Appl. Environ. Microbiol. 73:1576-1585.

Martínez, I., C. E. Muller, and J. Walter. 2013. Long-term temporal analysis of the human fecal microbiota revealed a stable core of dominant bacterial species. PLoS ONE 8:e69621.

McNulty, N. P., T. Yatsunenko, A. Hsiao, J. J. Faith, B. D. Muegge, A. L. Goodman, B. Henrissat, R. Oozeer, S. Cools-Portier, G. 
Gobert, C. Chervaux, D. Knights, C. A. Lozupone, R. Knight, A. E. Duncan, J. R. Bain, M. J. Muehlbauer, C. B. Newgard, A. C. Heath, and J. I. Gordon. 2011. The impact of a consortium of fermented milk strains on the gut microbiome of gnotobiotic mice and monozygotic twins. Sci. Transl. Med. 3:106ra106.

Momozawa, Y., V. Deffontaine, E. Louis, and J. F. Medrano. 2011. Characterization of bacteria in biopsies of colon and stools by high throughput sequencing of the V2 region of bacterial 16S rRNA gene in human. PLoS ONE 6:e16952.

Nam, Y. D., M. J. Jung, S. W. Roh, M. S. Kim, and J. W. Bae. 2011. Comparative analysis of Korean human gut microbiota by barcoded pyrosequencing. PLoS ONE 6:e22109.

Nawrocki, E. P., D. L. Kolbe, and S. R. Eddy. 2009. Infernal 1.0: Inference of RNA alignments. Bioinformatics 25:1335-1337.

Nikooyeh, B., T. R. Neyestani, M. Farvid, H. Alavi-Majd, A. Houshiarrad, A. Kalayi, N. Shariatzadeh, A. Gharavi, S. Heravifard, N. Tayebinejad, S. Salekzamani, and M. Zahedirad. 2011. Daily consumption of vitamin D- or vitamin D + calcium-fortified yogurt drink improved glycemic control in patients with type 2 diabetes: a randomized clinical trial. Am. J. Clin. Nutr. 93:764-771.

Osei-Poku, J., C. M. Mbogo, W. J. Palmer, and F. M. Jiggins. 2012. Deep sequencing reveals extensive variation in the gut microbiota of wild mosquitoes from Kenya. Mol. Ecol. 21:5138-5150.

Palaria, A., I. Johnson-Kanda, and D. J. O'Sullivan. 2012. Effect of a synbiotic yogurt on levels of fecal bifidobacteria, clostridia, and enterobacteria. Appl. Environ. Microbiol. 78:933-940.

Pepper, J. W., and S. Rosenfeld. 2012. The emerging medical ecology of the human gut microbiome. Trends Ecol. Evol. 27:381-384.

Price, M. N., P. S. Dehal, and A. P. Arkin. 2009. FastTree: Computing large minimum evolution trees with profiles instead of a distance matrix. Mol. Biol. Evol. 26:1641-1650.

Quince, C., A. Lanzen, R. J. Davenport, and P. J. Turnbaugh. 2011. Removing noise from pyrosequenced amplicons. BMC Bioinformatics 12:38

Rolfe, R. D. 2000. The role of probiotic cultures in the control of gastrointestinal health. J. Nutr. 130(2S Suppl.):396S-402S.

Sanz, Y., A. Santacruz, and G. De Palma. 2008. Insights into the roles of gut microbes in obesity. Interdiscip. Perspect. Infect. Dis. 2008:829101

Saxelin, M., A. Lassig, H. Karjalainen, S. Tynkkynen, A. Surakka, H. Vapaatalo, S. Jarvenpaa, R. Korpela, M. Mutanen, and K. Hatakka. 2010. Persistence of probiotic strains in the gastrointestinal tract when administered as capsules, yoghurt, or cheese. Int. J. Food Microbiol. 144:293-300.

Schiffrin, E. J., A. Parlesak, C. Bode, J. C. Bode, M. A. van't Hof, D. Grathwohl, and Y. Guigoz. 2009. Probiotic yogurt in the elderly with intestinal bacterial overgrowth: endotoxaemia and innate immune functions. Br. J. Nutr. 101:961-966.
Schloss, P. D., D. Gevers, and S. L. Westcott. 2011. Reducing the effects of PCR amplification and sequencing artifacts on 16S rRNAbased studies. PLoS ONE 6:e27310.

Schloss, P. D., and S. L. Westcott. 2011. Assessing and improving methods used in operational taxonomic unit-based approaches for $16 \mathrm{~S}$ rRNA gene sequence analysis. Appl. Environ. Microbiol. 77:3219-3226.

Schloss, P. D., S. L. Westcott, T. Ryabin, J. R. Hall, M. Hartmann, E. B. Hollister, R. A. Lesniewski, B. B. Oakley, D. H. Parks, C. J. Robinson, J. W. Sahl, B. Stres, G. G. Thallinger, D. J. Van Horn, and C. F. Weber. 2009. Introducing Mothur: Open-source, platform-independent, community-supported software for describing and comparing microbial communities. Appl. Environ. Microbiol. 75:7537-7541.

Shin, H. S., S. Y. Park, K. Lee Do, S. A. Kim, H. M. An, J. R. Kim, M. J. Kim, M. G. Cha, S. W. Lee, K. J. Kim, K. O. Lee, and N. J. Ha. 2010. Hypocholesterolemic effect of sonication-killed Bifidobacterium longum isolated from healthy adult Koreans in high cholesterol fed rats. Arch. Pharm. Res. 33:1425-1431.

Sim, K. M. J. Cox, H. Wopereis, R. Martin, J. Knol, M. S. Li, W. O. Cookson, M. F. Moffatt, and J. S. Kroll. 2012. Improved detection of bifidobacteria with optimised 16S rRNA-gene based pyrosequencing. PLoS ONE 7:e32543.

Sun, X., and M. B. Zemel. 2004. Calcium and dairy products inhibit weight and fat regain during ad libitum consumption following energy restriction in Ap2-agouti transgenic mice. J. Nutr. 134:3054-3060.

Tremaroli, V., and F. Backhed. 2012. Functional interactions between the gut microbiota and host metabolism. Nature 489:242-249.

Unno, T., J. Jang, D. Han, J. H. Kim, M. J. Sadowsky, O. S. Kim, J. Chun, and H. G. Hur. 2010. Use of barcoded pyrosequencing and shared OTUs to determine sources of fecal bacteria in watersheds. Environ. Sci. Technol. 44:7777-7782.

Vintiñi, E. O., and M. S. Medina. 2011. Host immunity in the protective response to nasal immunization with a pneumococcal antigen associated to live and heat-killed Lactobacillus casei. BMC Immunol. 12:46.

Wang, T., G. Cai, Y. Qiu, N. Fei, M. Zhang, X. Pang, W. Jia, S. Cai, and L. Zhao. 2012. Structural segregation of gut microbiota between colorectal cancer patients and healthy volunteers. ISME J. 6:320-329.

White, K. M., S. J. Bauer, K. K. Hartz, and M. Baldridge. 2009. Changes in body composition with yogurt consumption during resistance training in women. Int. J. Sport Nutr. Exerc. Metab. 19:18-33. 